\title{
Analysis of Classical and New Visual Servoing Control Laws
}

\author{
Mohammed Marey and François Chaumette
}

\begin{abstract}
In this paper, we analyze and compare five imagebased visual servoing control laws. Three of them are classical while two new ones are proposed. The first new control law is based on a behavior controller to adjust the movement of the camera. It can also be used to switch between the classical methods. The second control law is designed to try to obtain the global stability of the system. An analytical study of all control schemes when translational motion along and rotational motion around the optical axis is also presented. Finally, simulation and experimental results show that the new control law with a behavior controller has a wider range of success than the other control schemes and can be used to avoid local minima and singularities.
\end{abstract}

\section{INTRODUCTION}

Visual servoing is a well known approach to increase the accuracy, the versatility and the robustness of a visionbased robotic system [11], [5]. Two main aspects have a great impact on the behavior of any visual servoing scheme: the selection of the visual features used as input of the control law and the form of the control scheme. As for the visual features, they can be selected in the image space (point coordinates, parameters representing straight lines or ellipses, moments,... [8], [12], [6], [9], [4]), in the Cartesian space (pose, coordinates of 3D points,... [16], [17]), or composed of a mixture of both kinds of features attempting to incorporate the advantages of both image-based and positionbased methods [13], [7], [2]. As for the choice of the control law [8], [14], [5], it affects the behavior of the selected visual features (local or global exponential decrease, second order minimization, ...) and may lead, or not, to local minima and singularities [3].

This paper is not concerned with the choice of the visual features, but with the analysis of different control schemes. That is why we will consider the most usual and simple features, that are the Cartesian coordinates of image points. As for the control schemes, we consider three classical control laws and we also propose in this paper two new ones. The first new control law follows an hybrid strategy. It is based on a behavior parameter that can be used to tune the weight of the current and the desired interaction matrix in the control law. We will see that in some configurations where all other control schemes fail, this new control law allows the system to converge. The second control law that we propose is an attempt towards global asymptotic stability (GAS). Unfortunately, if GAS can be obtained in the space of the chosen task function, we will see that it is not ensured

Mohammed Marey and François Chaumette are with IRISA/INRIARennes, Campus de Beaulieu, 35042 Rennes, France. E-mail: Firstname.Namedirisa.fr

Mohammed Marey is granted by the Egyptian Government. in the configuration space $S E_{3}$ when redundant image point coordinates are used. This control scheme is indeed subject to attractive local minima. The paper also includes an analysis of the control laws with respect to translational motion along and rotational motion around the optical axis. As we will see, a singularity of the control law proposed in [14] will be exhibited thanks to this analysis.

The paper is organized as follows: in Section II, classical control schemes are recalled from which the control law with a behavior controller is proposed. In Section III, another control law is proposed and it global stability is studied. In Section IV, an analysis of the control laws in the presence of rotation and translation w.r.t. the camera optical axis is presented. Finally, simulation and experimental results are presented in Section V.

\section{NEW CONTROLLER WITH A BEHAVIOR PARAMETER}

Let $\mathbf{s} \in \mathbb{R}^{k}$ be the vector of the selected $k$ visual features, $\mathbf{s}^{*}$ their desired value and $\mathbf{v} \in \mathbb{R}^{6}$ the instantaneous velocity of the camera. Most classical control laws have the following form:

$$
\mathbf{v}=-\lambda \widehat{\mathbf{L}}_{\mathbf{s}}^{+}\left(\mathbf{s}-\mathbf{s}^{*}\right)
$$

where $\lambda$ is a gain and $\widehat{\mathbf{L}}_{\mathbf{s}}^{+}$is the pseudoinverse of an estimation or an approximation of the interaction matrix related to $\mathbf{s}$ (defined such that $\dot{\mathbf{s}}=\mathbf{L}_{\mathbf{s}} \mathbf{v}$ where $\mathbf{v}=(\boldsymbol{v}, \boldsymbol{\omega})$ with $v$ the translational velocity and $\boldsymbol{\omega}$ the rotational one). Different forms for $\widehat{\mathbf{L}_{\mathbf{s}}}$ have been proposed in the past [8], [5]. For simplicity, we consider that all values can be computed accurately, leading to the following choices

$$
\begin{aligned}
& 1): \widehat{\mathbf{L}_{\mathbf{s}}}=\mathbf{L}_{\mathbf{s}^{*}} \\
& 2): \widehat{\mathbf{L}_{\mathbf{s}}}=\mathbf{L}_{\mathbf{s}(t)} \\
& 3): \widehat{\mathbf{L}_{\mathbf{s}}}=\left(\mathbf{L}_{\mathbf{s}^{*}}+\mathbf{L}_{\mathbf{s}(t)}\right) / 2 .
\end{aligned}
$$

In the first case, $\widehat{\mathbf{L}_{\mathbf{s}}}$ is constant during all the servo since it is the value of the interaction matrix computed at the desired configuration. In the second case, $\widehat{\mathbf{L}_{\mathrm{s}}}$ changes at each iteration of the servo since the current value of the interaction matrix is used. Finally, in the third case, the average of these two values is used [14]. These three usual choices for $\widehat{\mathbf{L}_{\mathrm{s}}}$ when used with (1) define three distinct control laws, that we will denote $\mathrm{D}, \mathrm{C}$ and $\mathrm{A}$ (for desired, current and average respectively) in the remainder of the paper.

On one hand, near the desired pose where the error $\mathbf{s}-\mathbf{s}^{*}$ is low, the same behavior is obtained whatever the choice of $\widehat{\mathbf{L}_{\mathbf{s}}}$ since we have in that case $\mathbf{L}_{\mathbf{s}(t)} \approx \mathbf{L}_{\mathbf{s}^{*}}$. On the other hand, as soon as $\mathbf{s}-\mathbf{s}^{*}$ is large, it is well known that the choice of $\widehat{\mathbf{L}_{\mathrm{s}}}$ induces a particular behavior of the system 
since we thus have $\mathbf{L}_{\mathbf{s}(t)} \neq \mathbf{L}_{\mathbf{s}^{*}}$. This motivates the current research on the determination of visual features such that the interaction matrix is constant in all the configuration space of the camera, but it is clearly still an open problem, and, as already said, not the subject of this paper.

From (2), (3) and (4), a general form for $\widehat{\mathbf{L}_{\mathbf{s}}}$ can easily be written by introducing a behavior controller $\beta \in \mathbb{R}$

$$
\widehat{\mathbf{L}_{\mathbf{s}}}=\mathbf{L}_{\beta}=\left(\beta \mathbf{L}_{\mathbf{s}^{*}}+(1-\beta) \mathbf{L}_{\mathbf{s}(t)}\right) \text {. }
$$

Using (5) in (1), we obtain a new control law, denoted $G$ in the following (for "general"). Of course, if $\beta=1$, we find again control law $\mathrm{D}$, if $\beta=0$, we obtain control law $\mathrm{C}$, and if $\beta=1 / 2$ we obtain control law $A$. Control law $\mathrm{G}$ could thus be used to switch between the different control schemes during the execution of the task. Switching strategies have already been proposed in [10], [1] but, in these works, switching is performed between image-based and positionbased approaches, that is between different features, while here the features are the same but their control would be different.

In this paper, we are not interested in designing a possible strategy to switch between the different control laws. We are looking if particular values of $\beta$ provide a better behavior of the system. Indeed, the main interesting property of control law $G$ is that the behavior of the system changes gradually from the behavior using control law $\mathrm{C}$ to the behavior using control law A when $\beta$ varies from 0 to $1 / 2$, and similarly, the behavior changes gradually from the behavior using control law $\mathrm{A}$ to the behavior using control law $\mathrm{D}$ when $\beta$ varies from $1 / 2$ to 1 . Hence, this new control scheme allows us to adapt the behavior of the system based on the selected value of $\beta$. We will see in Section $\mathrm{V}$ that particular values of $\beta$ indeed allow the system to converge while the other control schemes fail.

Let us finally note that in case of modeling or calibration errors, the matrices $\mathbf{L}_{\mathbf{s}^{*}}$ and $\mathbf{L}_{\mathbf{s}(t)}$ have to be respectively replaced by approximations $\widehat{\mathbf{L}_{\mathbf{s}^{*}}}$ and $\widehat{\mathbf{L}_{\mathbf{s}(t)}}$, but that does not change the general properties of the control schemes as long as the approximations are not too coarse.

\section{PSEUDO-GAS CONTROL LAW}

Control laws D, C, and A are known to be locally asymptotically stable only [5]. The same is of course true for control law G. In this section, an attempt to obtain a GAS control scheme is presented.

\section{A. Modeling}

Let us choose as task function $\mathbf{e} \in \mathbb{R}^{\mathbf{6}}$ the following error

$$
\mathbf{e}=\mathbf{L}_{\mathbf{s}^{*}}^{+}\left(\mathbf{s}-\mathbf{s}^{*}\right)
$$

where, as usual, $\mathbf{s}^{*}$ is chosen such that $\mathbf{L}_{\mathbf{s}^{*}}$ is a full rank matrix. Since $\mathbf{L}_{\mathbf{s}^{*}}$ is constant, the time variation of $\mathbf{e}$ is given by $\dot{\mathbf{e}}=\mathbf{L}_{\mathbf{e}} \mathbf{v}$ where $\mathbf{L}_{\mathbf{e}} \in \mathbb{R}^{6 \times 6}$ is given by

$$
\mathbf{L}_{\mathbf{e}}=\mathbf{L}_{\mathbf{s}^{*}}^{+} \mathbf{L}_{\mathbf{s}}
$$

We can note that $\mathbf{L}_{\mathbf{e}}$ is of full rank 6 as soon as $\mathbf{L}_{\mathbf{s}}$ is also of full rank 6. To achieve an exponential decreasing of $\mathbf{e}$ (that is, $\dot{\mathbf{e}}=-\lambda \mathbf{e}$ ), we obtain immediately as control scheme

$$
\mathbf{v}=-\lambda \mathbf{L}_{\mathbf{e}}^{-1} \mathbf{e}
$$

which is nothing but

$$
\mathbf{v}=-\lambda\left(\mathbf{L}_{\mathbf{s}^{*}}^{+} \mathbf{L}_{\mathbf{s}}\right)^{-1} \mathbf{L}_{\mathbf{s}^{*}}^{+}\left(\mathbf{s}-\mathbf{s}^{*}\right) .
$$

\section{B. Stability analysis}

To study the stability of the control scheme (8), let us consider as candidate Lyapunov function $\mathcal{L}=\frac{1}{2}\|\mathbf{e}(t)\|^{2}$. We have $\dot{\mathcal{L}}=\mathbf{e}^{\top} \dot{\mathbf{e}}=\mathbf{e}^{\top} \mathbf{L}_{\mathbf{e}} \mathbf{v}$. Applying (7), we obtain

$$
\begin{aligned}
\dot{\mathcal{L}} & =-\lambda \mathbf{e}^{\top} \mathbf{L}_{\mathbf{e}} \mathbf{L}_{\mathbf{e}}^{-1} \mathbf{e}=-\lambda \mathbf{e}^{\top} \mathbf{e} \\
& <0, \forall \mathbf{e} \neq 0 .
\end{aligned}
$$

The control scheme (8) seems thus to be very promising since it is GAS in the task function space. Indeed, $\mathcal{L}$ always decrease to 0 whatever the initial value of e. Furthermore, e ensures the specified behavior $\dot{\mathbf{e}}=-\lambda \mathbf{e}$ as soon as $\mathbf{L}_{\mathbf{s}^{*}}$ and $\mathbf{L}_{\mathbf{s}}$ are computed accurately. Unfortunately, to end the demonstration of the global stability, we should demonstrate that $\mathbf{e}=0$ if and only if $\mathbf{s}=\mathbf{s}^{*}$. That is usually impossible since, as soon as

$$
\left(\mathbf{s}-\mathbf{s}^{*}\right) \in \operatorname{Ker} \mathbf{L}_{\mathbf{s}^{*}}^{+},
$$

we have $\mathbf{e}=0$, which implies $\mathbf{v}=0$, and $\mathbf{s} \neq \mathbf{s}^{*}$, which corresponds to a local minimum [3]. In other words, GAS in the task function space does not necessarily implies GAS in $S E_{3}$ or in the visual features space. The task function (6) forms a local diffeomorphism with $S E_{3}$, but not a global one as soon as a configuration such that (9) is satisfied exists. Control law (8) will thus be denoted PG in the following (for "pseudo-GAS").

In Section V, we will exhibit some configurations which lead to local minima. We can thus conclude that, as for all the previous control schemes, only the local asymptotic stability of PG can be demonstrated when image point coordinates are used as visual features. In spite of this disappointing result, control scheme PG is still interesting, since it may be possible in the future to determine visual features such that $\operatorname{Ker} \mathbf{L}_{\mathbf{s}^{*}}^{+}=\mathbf{0}$, leading to GAS.

\section{Motion ALONG AND AROUND THE OPTICAL AXIS}

This section presents an analytical analysis of all the control laws described previously when the camera displacement is a combination of a translation $t_{z}$ and a rotation $r_{z}$ w.r.t. the camera optical axis. As usually done in IBVS, we have considered an object composed of four points forming a square.

The study includes two cases in which the movement along $\mathrm{z}$-axis is from $Z$ to $Z^{*}$ and where $r_{z}=90^{\circ}$ in the first case and $r_{z}=180^{\circ}$ in the second case. In both cases, the object plane is parallel to the image plane.

The coordinates of a 3D point in the camera frame are denoted $(X, Y, Z)$ and the coordinates of that point on the image plane are given by $\mathbf{x}=(x, y)$ with $x=X / Z$ and 
$y=Y / Z$. It is well known that the interaction matrix related to $\mathrm{x}$ is given by

$$
\mathbf{L}_{\mathbf{x}}=\left[\begin{array}{cccccc}
\frac{-1}{Z} & 0 & \frac{x}{Z} & x y & -\left(1+x^{2}\right) & y \\
0 & \frac{-1}{Z} & \frac{y}{Z} & 1+y^{2} & -x y & -x
\end{array}\right]
$$

Using four points, the visual feature is defined by $\mathbf{s}=$ $\left(x_{0}, x_{1}, x_{3}, x_{4}, y_{0}, y_{1}, y_{2}, y_{3}\right)$ whose desired value is $\mathbf{s}^{*}=$ $\left(x_{0}^{*}, x_{1}^{*}, x_{2}^{*}, x_{3}^{*}, y_{0}^{*}, y_{1}^{*}, y_{2}^{*}, y_{3}^{*}\right)$.

1) Case 1: $r_{z}=90^{\circ} \& t_{z}=\left(Z \rightarrow Z^{*}\right)$ : The coordinates of the four points w.r.t. the camera frame at the initial and the desired poses are denoted $p_{i 0}=(-L,-L, Z)$, $p_{i 1}=(-L, L, Z), p_{i 2}=(L, L, Z), p_{i 3}=(L,-L, Z)$, $p_{d 0}=\left(-L, L, Z^{*}\right), p_{d 1}=\left(L, L, Z^{*}\right), p_{d 2}=\left(L,-L, Z^{*}\right)$ and $p_{d 3}=\left(-L,-L, Z^{*}\right)$. Let $l=L / Z$ and $l^{*}=L / Z^{*}$. The initial value of $\mathbf{s}$ is then $\mathbf{s}_{\mathbf{i}}=(-l,-l, l, l,-l, l, l,-l)$, the desired value is $\mathbf{s}^{*}=\left(-l^{*}, l^{*}, l^{*},-l^{*}, l^{*}, l^{*},-l^{*},-l^{*}\right)$ and $\mathbf{s}_{\mathbf{i}}-\mathbf{s}^{*}=\left(-l+l^{*},-l-l^{*}, l^{*}-l, l+l^{*},-l-l^{*}, l-l^{*}, l+\right.$ $\left.l^{*}, l^{*}-l\right)$ is the error vector. Using the analytical form of $\mathbf{L}_{\mathbf{x}}$, it is possible to compute the analytical form of $\mathbf{L}_{\beta}$ defined in (5) and then its pseudoinverse $\mathbf{L}_{\beta}^{+}$. Using $Z=l^{*} Z^{*} / l$, we obtain after computations and simplifications

$$
\mathbf{L}_{\beta}^{+}=\left[\begin{array}{cccccccc}
-c_{0} & -c_{0} & -c_{0} & -c_{0} & -c_{1} & c_{1} & -c_{1} & c_{1} \\
-c_{1} & c_{1} & -c_{1} & c_{1} & -c_{0} & -c_{0} & -c_{0} & -c_{0} \\
-c_{3} & c_{4} & c_{3} & -c_{4} & c_{4} & c_{3} & -c_{4} & -c_{3} \\
-c_{5} & c_{5} & -c_{5} & c_{5} & 0 & 0 & 0 & 0 \\
0 & 0 & 0 & 0 & c_{5} & -c_{5} & c_{5} & -c_{5} \\
c_{7} & c_{6} & -c_{6} & -c_{7} & c_{6} & -c_{7} & -c_{6} & c_{7}
\end{array}\right]
$$

where, when $\beta \in[0 ; 1]$,

$$
\begin{aligned}
& c_{0}=\frac{l^{*} Z^{*}}{4\left(\beta l^{*}+(1-\beta) l\right)} \\
& c_{1}= \begin{cases}0 & \text { if } \beta l^{* 2}=(1-\beta) l^{2} \\
c_{0} \frac{\beta\left(1+l^{* 2}\right)+(1-\beta)\left(1+l^{2}\right)}{\left(\beta l^{* 2}-(1-\beta) l^{2}\right)} & \text { else. }\end{cases} \\
& c_{3}=\frac{l^{*} Z^{*}\left(\beta l^{*}+(1-\beta) l\right)}{8\left((1-\beta)^{2} l^{3}+\beta^{2} l^{* 3}\right)}, c_{4}=\frac{l^{*} Z^{*}\left(\beta l^{*}-(1-\beta) l\right)}{8\left((1-\beta)^{2} l^{3}+\beta^{2} l^{* 3}\right)} \\
& c_{5}= \begin{cases}0 & \text { if } \beta l^{*} \\
\frac{-1}{4\left(\beta l^{* 2}-(1-\beta) l^{2}\right)} & \text { else. }\end{cases} \\
& c_{6}=\frac{\beta l^{* 2}+(1-\beta) l^{2}}{8\left((1-\beta)^{2} l^{3}+\beta^{2} l^{* 3}\right)}, c_{7}=\frac{\beta l^{* 2}-(1-\beta) l^{2}}{8\left((1-\beta)^{2} l^{3}+\beta^{2} l^{* 3}\right)}
\end{aligned}
$$

Using the value of $\mathbf{s}_{\mathbf{i}}-\mathbf{s}^{*}$, the initial velocity $\mathbf{v}_{\mathbf{i}}$ is easily deduced from (1) as

$$
\mathbf{v}_{\mathbf{i}}=\left(0,0, v_{z}, 0,0, \omega_{z},\right)
$$

where

$v_{z}=\frac{\lambda Z^{*} l^{*}\left(\beta l^{* 2}-(1-\beta) l^{2}\right)}{\beta^{2} l^{*}+(1-\beta)^{2} l^{3}}, \omega_{z}=\frac{\lambda l l^{*}\left(\beta l^{*}+(1-\beta) l\right)}{\beta^{2} l^{*^{3}}+(1-\beta)^{2} l^{3}}$

As expected, the initial camera motion consists in performing a translation combined with a rotation whose value only depends on image data and on the chosen value for $\beta$ and $\lambda$. We can note that $\mathbf{L}_{\beta}$ is singular if $\beta l^{* 2}=(1-\beta) l^{2}$. For instance, such a singularity occurs when $l=l^{*}$ (i.e. $Z=Z^{*}$ ) and $\beta=1 / 2$, which is very surprising. The control law A proposed in [14] is thus singular for a pure rotation of $90^{\circ}$, which had not been exhibited before as far as we know. In fact, the only way to avoid this singularity whatever the value of $l$ and $l^{*}$ is to select $\beta=0$ or $\beta=1$. As can be seen on (10), this singularity has no effect on the computed velocity in perfect conditions, but, as we will see in Section $\mathrm{V}$, a quite unstable behavior is obtained in the presence of image noise or for configurations near that singularity (such that for instance the object plane is almost parallel to the image plane).

When $Z=Z^{*}$ then $l=l^{*}$ and the initial velocity $\mathbf{v}_{\mathbf{i}}$ becomes

$$
\mathbf{v}_{\mathbf{i}}=\left(0,0, \frac{\lambda Z^{*}(2 \beta-1)}{2 \beta^{2}-2 \beta+1}, 0,0, \frac{\lambda}{2 \beta^{2}-2 \beta+1}\right) .
$$

In that classical case, the velocity $\mathbf{v}_{\mathbf{i}}$ contains an unexpected translation whose direction depends on the value of $\beta\left(v_{z}<\right.$ 0 if $\beta<1 / 2$ and $v_{z}>0$ if $\beta>1 / 2$ ). The only way to avoid this nonzero translation is to select $\beta=1 / 2$ as already shown in [14], but $\mathbf{L}_{\beta}$ is singular in that case...

Coming back to the more general case and setting $\beta=1$ in $\mathbf{L}_{\beta}^{+}$, the initial velocity $\mathbf{v}_{\mathbf{i}}$ using control law $\mathrm{D}$ is given by

$$
\mathbf{v}_{\mathbf{i}}=\left(0,0, \lambda Z^{*}, 0,0, \frac{\lambda l}{l^{*}}\right) .
$$

Whatever the value of $Z$, that is even when $Z<Z^{*}$ in which case the camera has to move backward, the initial camera motion contains a forward translational term. This surprising result extends the same property obtained when $Z=Z^{*}[5]$.

Setting $\beta=0$, the initial velocity $\mathbf{v}_{\mathbf{i}}$ using the control law $\mathrm{C}$ is now

$$
\mathbf{v}_{\mathbf{i}}=\left(0,0, \frac{-\lambda l^{*} Z^{*}}{l}, 0,0, \frac{\lambda l^{*}}{l}\right) .
$$

In that case, the initial camera motion contains a backward translational term whatever the value of $Z$, that is even when $Z \geq Z^{*}$. We can even note that, more $l$ is small, i.e. more $Z$ is large, more the initial backward motion is large, which is even more surprising than the result obtained for $\beta=1$. These results extend thus largely the property exhibited in [6] when $Z=Z^{*}$. By comparing (11) and (12), we can also note that the amplitude of the rotational motion using control laws $\mathrm{D}$ and $\mathrm{C}$ is surprisingly not the same as long as $l \neq l^{*}$, that is as soon as $Z \neq Z^{*}$.

Setting $\beta=1 / 2$, the velocity $\mathbf{v}_{\mathbf{i}}$ using control law $\mathrm{A}$ is

$$
\mathbf{v}_{\mathbf{i}}=\left(0,0, \frac{2 \lambda Z^{*} l^{*}\left(l^{* 2}-l^{2}\right)}{l^{* 3}+l^{3}}, 0,0, \frac{2 \lambda l l^{*}\left(l+l^{*}\right)}{l^{3}+l^{* 3}}\right) .
$$

In that case, a good behavior is obtained since the translational motion is always in the expected direction $\left(v_{z}<0\right.$ when $l^{*}<l$, that is when $Z<Z^{*}, v_{z}>0$ when $l^{*}>l$ $\left(Z>Z^{*}\right.$ ), and, as already said, $v_{z}=0$ when $l=l^{*}$ (where $Z=Z^{*}$ but where $\widehat{\mathbf{L}_{\beta}}$ is singular).

Finally, the velocity $\mathbf{v}_{\mathbf{i}}$ of the new control law PG is

$$
\mathbf{v}_{\mathbf{i}}=\left(0,0, \frac{-\lambda l^{*} Z^{*}}{l}, 0,0, \frac{\lambda l^{*}}{l}\right)
$$

that is exactly the same velocity $\mathbf{v}_{\mathbf{i}}$ given in (12) by the control law $\mathrm{C}$.

2) Case 2: $r_{z}=180^{\circ} \& t_{z}=\left(Z \rightarrow Z^{*}\right)$ : We now consider the more problematic case where the camera displacement is composed of a translation and of a rotation of $180^{\circ}$ around the camera optical axis. In that case, $\mathbf{s}_{\mathbf{i}}-\mathbf{s}^{*}=$ $\left(l+l^{*},-l-l^{*},-l-l^{*}, l+l^{*},-l-l^{*},-l-l^{*}, l+l^{*}, l+l^{*}\right)$ 
and $\mathbf{L}_{\beta}^{+}$is given by

$$
\mathbf{L}_{\beta}^{+}=\left[\begin{array}{cccccccc}
-c_{0} & -c_{0} & -c_{0} & -c_{0} & -c_{1} & c_{1} & -c_{1} & c_{1} \\
-c_{1} & c_{1} & -c_{1} & c_{1} & -c_{0} & -c_{0} & -c_{0} & -c_{0} \\
-c_{3} & c_{3} & c_{3} & -c_{3} & c_{3} & c_{3} & -c_{3} & -c_{3} \\
-c_{4} & c_{4} & -c_{4} & c_{4} & 0 & 0 & 0 & 0 \\
0 & 0 & 0 & 0 & c_{4} & -c_{4} & c_{4} & -c_{4} \\
c_{5} & c_{5} & -c_{5} & -c_{5} & c_{5} & -c_{5} & -c_{5} & c_{5}
\end{array}\right]
$$

where, when $\beta \in[0 ; 1]$,

$$
\begin{aligned}
& c_{0}=\frac{l^{*} Z^{*}}{4\left(\beta l^{*}+(1-\beta) l\right)}, c_{1}=c_{0} \frac{\beta\left(1+l^{* 2}\right)+(1-\beta)\left(1+l^{2}\right)}{\beta l^{* 2}+(1-\beta) l^{2}} \\
& c_{3}= \begin{cases}0 & \text { if } \beta l^{* 2}=(1-\beta) l^{2} \\
\frac{l^{*} Z^{*}}{8\left(\beta l *^{2}-(1-\beta) l^{2}\right)} & \text { else }\end{cases} \\
& c_{4}=\frac{1}{4\left(\beta l^{* 2}+(1-\beta) l^{2}\right)} \\
& c_{5}= \begin{cases}0 & \text { if } \beta l^{*}=(1-\beta) l \\
\frac{1}{8\left(\beta l^{*}-(1-\beta) l\right)} & \text { else }\end{cases}
\end{aligned}
$$

Proceeding as before, we obtain using the value of $\mathbf{s}_{\mathbf{i}}-\mathbf{s}^{*}$

$$
\begin{gathered}
\mathbf{v}_{\mathbf{i}}=\left(0,0, v_{z}, 0,0,0,\right) \\
\text { where } v_{z}= \begin{cases}0 & \text { if } \beta l^{* 2}=(1-\beta) l^{2} \\
\frac{\lambda Z^{*} l^{*}\left(l+l^{*}\right)}{\beta l^{* 2}-(1-\beta) l^{2}} & \text { else. }\end{cases}
\end{gathered}
$$

In all cases, no rotational motion is produced while a translational motion is generally obtained, but when $\beta l^{* 2}=$ $(1-\beta) l^{2}$ in which case $\mathbf{L}_{\beta}$ is singular, leading to a repulsive local minimum where $v_{z}=0$. Such a case occurs for instance when $Z=Z^{*}$ (i.e. $l=l^{*}$ ) and $\beta=1 / 2$, which corresponds to the control law proposed in [14]. Another singularity occurs when $\beta l^{*}=(1-\beta) l$, which is also the case when $l=l^{*}$ and $\beta=1 / 2$.

Of course, when $Z=Z^{*}$, we find again the results given in [3]: a pure forward motion is involved when $\beta=1$ and a pure backward motion is involved when $\beta=0$. More generally, for $\beta=1$ and $\beta=0$, the direction of motion is the same (i.e. forward or backward) whatever the value of $l$ and $l^{*}$, that is whatever the value of $Z$ with respect to $Z^{*}$. For any other value of $\beta$, the direction of motion depends on the relative value of $Z$ with respect to $Z^{*}$, but unfortunately, there does not exist any value of $\beta$ that will give a good behavior in that case since no rotational motion is computed by the control law. Finally, no better results are obtained using control law PG since we have in that case

$$
\mathbf{v}_{\mathbf{i}}=\left(0,0, \frac{-\lambda l^{*} Z^{*}\left(l+l^{*}\right)}{l^{2}}, 0,0,0\right)
$$

which is the same as the one obtained when $\beta=0$.

We will validate the results obtained in this section through experimental results presented at the end of the next section.

\section{Results}

In this section, simulation and experimental results are given. They have been obtained using the ViSP library [15] in which the new control schemes have been implemented.

\section{A. Simulation results}

First, we consider two difficult configurations and compare the results obtained with the different control schemes described previously. A pose is denoted as $\mathbf{p}=(\mathbf{t}, \mathbf{r})$ where $\mathbf{t}$ is the translation expressed in meter and $\mathbf{r}$ the roll, pitch and yaw angles expressed in degrees.
1) Case 1: The desired camera pose is $(0,0,0.5,0,0,0)$, which means that the camera has to be at $0.5 \mathrm{~m}$ in front of the square and such that the square appears as a centered square in the image. In this case, the configurations where $\left(\mathbf{s}-\mathbf{s}^{*}\right) \in \operatorname{Ker} \mathbf{L}_{\mathbf{s}^{*}}^{+}$correspond to very particular cases where the four points are aligned in the image [3]. The initial camera pose is $(0,0,0.4,80,20,10)$ and has thus a very different orientation than the desired one. The simulation results for the control laws D, C, A and PG are depicted on Fig. 1. Classical schemes D, C and A lead the camera to converge to its desired pose while, using the new control law PG, the camera reaches a configuration where the four points are aligned in the image. In fact, such local minima are attractive for PG while they are not for all other control schemes. As expected, the task function e defined in (6) converges exponentially to zero as shown in Fig. 3.a, but that is not sufficient to obtain a good behavior of the system... Finally, we have checked with additional simulations that control law $\mathrm{G}$ converges to the desired configuration for any value of $\beta \in[-1.9 ; 1.04]$ (see Fig. 1.e where the result for $\beta=0.4$ is given). It is thus not necessary that $\beta \in[0 ; 1]$ and negative values can even be chosen. For this configuration, the value of $\beta$ is thus not a crucial issue.

2) Case 2: The desired camera pose is now given by $(0,0,1,45,-30,30)$ which means that the desired position of the image plane is not parallel to the object. The initial camera pose is given by $(0,0,1,-46,30,30)$. As can be seen on Fig. 2.a, using control law D, the camera is first motionless, as in a local minimum, and then starts to diverge so that the points leave the camera field of view. Even if we do not consider this constraint (we are here in simulation where an image plane of infinite size can be assumed), the camera then reaches the object plane where $Z=0$, leading of course to a failure. From the results depicted in Fig. 2.b, 2.c, and 2.d, we can see that control laws C, A, and PG all fail in a local minimum. For PG, we can note once again that the task function e converges exponentially to zero as shown in Fig. 3.b. As for control law A, it is the first time, as far as we know, that such a local minimum problem has been exhibited. Finally, control law G is the only one to converge to the desired position as soon as $0.515<\beta<0.569$ (see Fig. 2.e). The oscillations observed in the camera velocity and in the visual features allow the camera to go out from the workspace corresponding to the attractive area of the local minimum for the other control schemes.

\section{B. Experimental results}

The experimental results have been obtained on a six degrees of freedom robot. They allow to validate the analysis presented in Section IV about the motion along and around the optical axis. The required camera motion is composed of a rotation of $170^{\circ}$ around the optical axis combined with a translation of $0.5 \mathrm{~m}$ along the optical axis toward the object (a square once again). As usual, gain $\lambda$ has been set to 0.1 .

As expected unfortunately, control law D makes the points leave the camera field of view due to a forward motion, while control laws $\mathrm{C}$ and $\mathrm{PG}$ make the robot reach its joints 

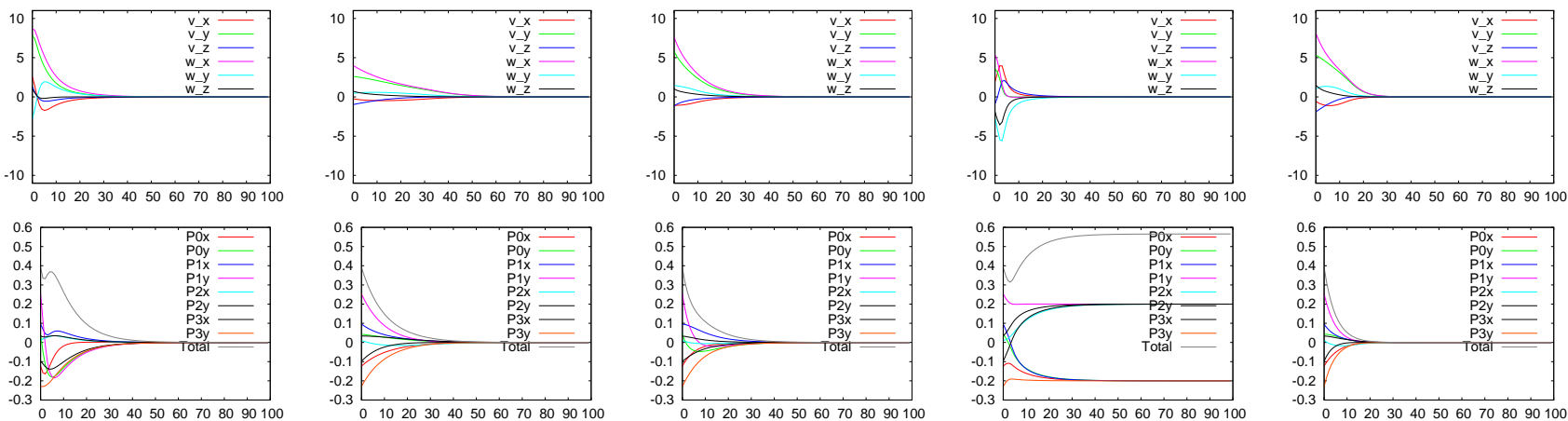

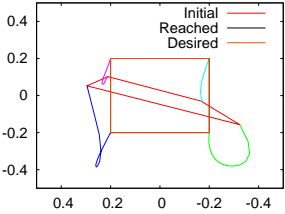

(a) control law D

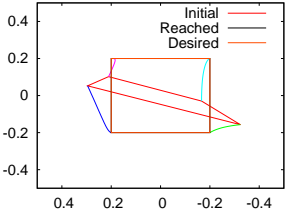

(b) control law C

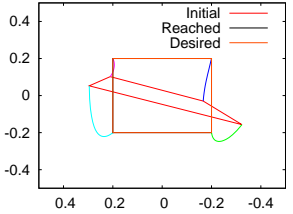

(c) control law A

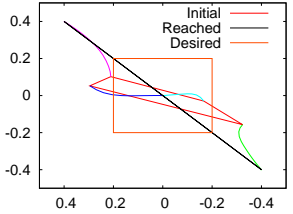

(d) control law PG

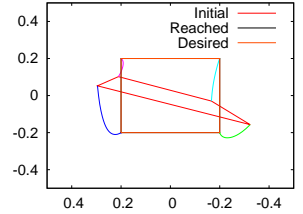

(e) control law $\mathrm{G}, \beta=0.4$

Fig. 1. Results for case 1. First line: camera velocity (in $\mathrm{m} / \mathrm{s}$ and $\mathrm{rad} / \mathrm{s}$ ), second line: image points error, third line: image points trajectories
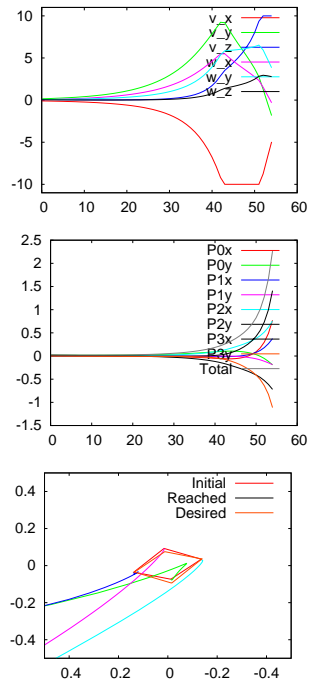

(a) control law D
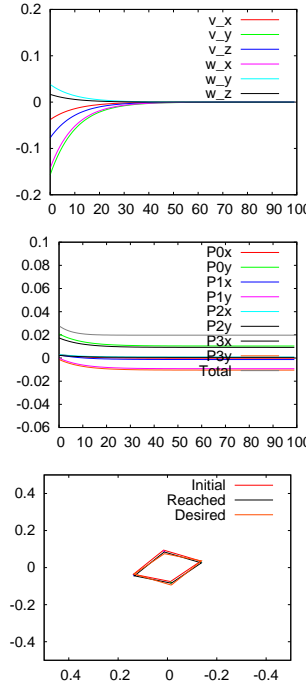

(b) control law C
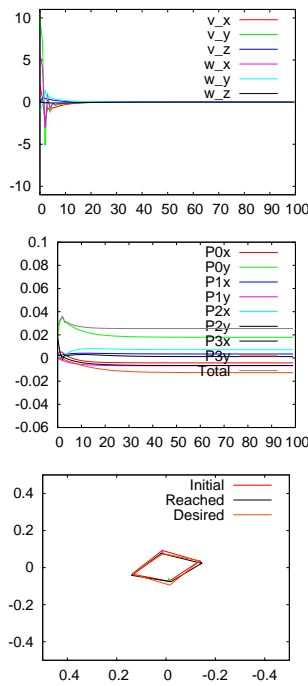

(c) control law A
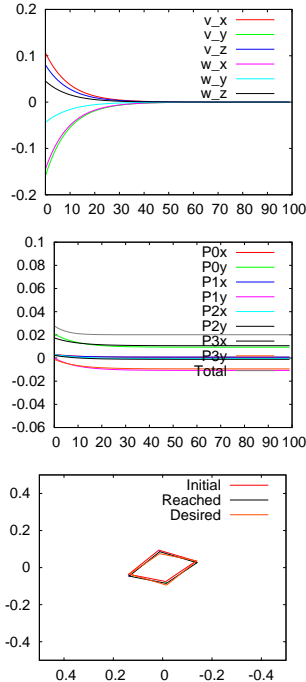

(d) control law PG
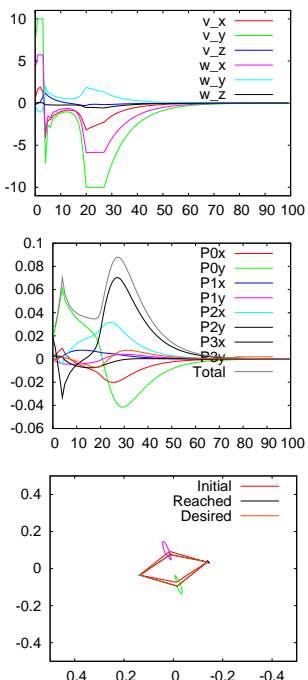

(e) control law G, $\beta=0.54$

Fig. 2. Results for case 2
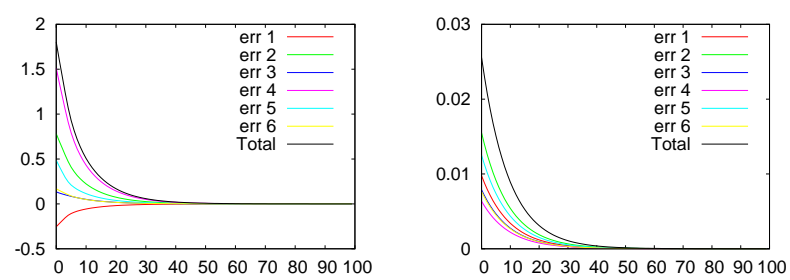

Fig. 3. Task function e for control law PG for case 1 (on the left) and case 2 (on the right)

limits due to a backward motion. As can be seen in Fig. 4.a, control law A starts with high value of $v_{z}$ toward the object, while $\omega_{z}$ increases until the translational motion is almost finished. Since the pure rotation $r_{z}=90^{\circ}$ corresponds to a singularity of control law A, as demonstrated in the analytical study, the behavior of the camera is quite unstable near this configuration, that is during 400 iterations (from iterations
800 to 1200) as can be observed in the velocity components in Fig. 4.a. As can be seen in Fig. 4.b, using control law $G$ with $\beta=0.4$ allows to decrease significantly the effect of the singularity near $r_{z}=90^{\circ}$, while its effect completely disappears for $\beta=0.35$ (see Fig. 4.c).

\section{CONCLUSIONS AND FUTURE WORK}

The control laws used in image-based visual servoing have their respective drawbacks and strengths. In some cases, a control law is not able to converge while the others succeed. In other cases, all classical control laws may fail. Different behaviors may explain these failures. For example, the camera moves to infinity, the camera moves to be too near to the object, the camera reaches a local minimum or a singular configuration. In this paper, new configurations have been exhibited, for the first time as far as we know: a local minimum for all classical control schemes, especially 

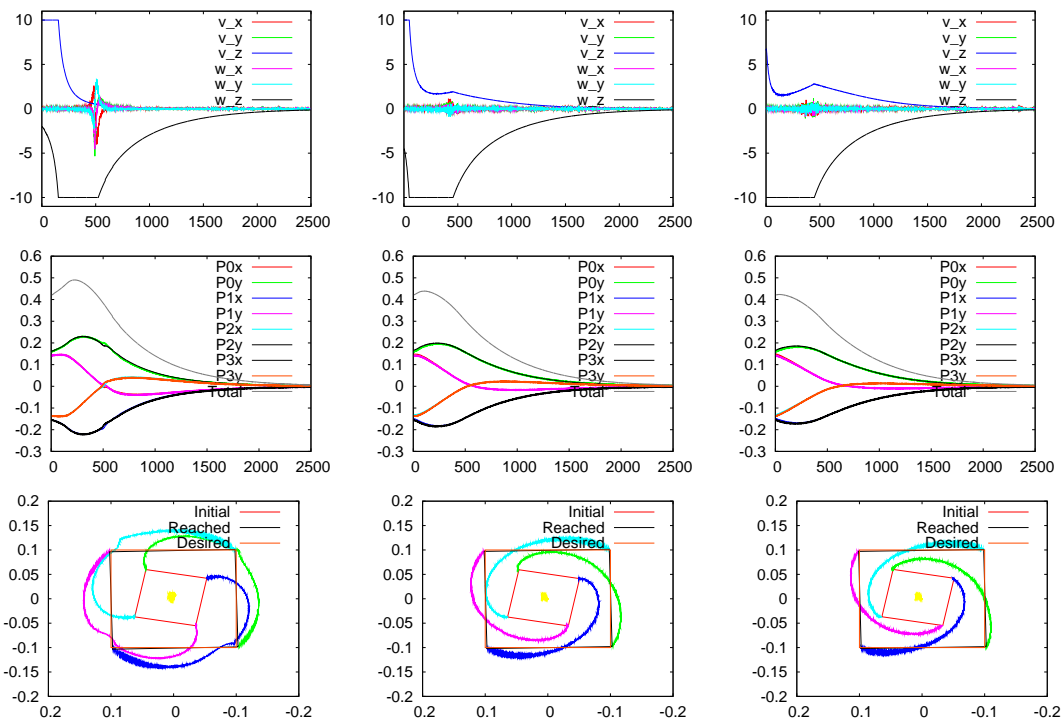

(a) control law $\mathrm{A}$

(b) control law G, $\beta=0.4$

(c) control law $\mathrm{G}, \beta=0.35$

Fig. 4. Experimental results for $r_{z}=170^{\circ}$ and $t_{z}=0.5 \mathrm{~m}$.

for the control law proposed in [14]. This configuration has been found by studying a new control scheme built to try to obtain its global asymptotic stability. A singularity of the control scheme proposed in [14] has also been exhibited and its effects have been emphasized through experiments obtained on a 6 dof robot. New surprising results have also been obtained for the other classical control schemes for motion combining translation along and rotation around the optical axis. Finally, a new control law based on a behavior controller has also been proposed. Setting $\beta=0,1$, or $1 / 2$ would allow to switch between the three most classical schemes but we have prefered to analyse the behavior of the control scheme for all possible values of this parameter. In all considered cases (difficult configurations subject to local minima for all classical schemes, motion along and around the optical axis), it has always been possible to determine values of this parameter that provide a satisfactory behavior of the control scheme. In fact, the suitable values of the behavior controller rely on the displacement that the camera has to realize. Future work will thus be devoted to determining how to select automatically the value of the behavior controller to obtain a good behavior in all cases. Modifying on line the value of the behavior controller during the task execution will be also studied.

\section{ACKNOWLEGMENTS}

The authors would like to thank Seth Hutchinson for his comments on this research and earlier draft of this paper.

\section{REFERENCES}

[1] A. H. Abdul Hafez, C.V. Jawahar, "Visual servoing by optimization of a 2D/3D hybrid objective function", ICRA'07, pp. 1691-1696, Roma, Apr. 2007.

[2] E. Cervera, A. Pobil, F. Berry, P. Martinet, "Improving imagebased visual servoing with three-dimensional features", Int. Journal of Robotics Research, 22:821-839, Oct. 2003.

[3] F. Chaumette, "Potential problems of stability and convergence in image-based and position-based visual servoing", The Conference of Vision and Control. LNCIS 237, pp 66-78, 1998.
[4] F. Chaumette, "Image moments: a general and useful set of features for visual servoing", IEEE Trans. on Robotics and Automation, 20(4):713723, Aug. 2004.

[5] F. Chaumette, S. Hutchinson, "Visual servo control Part I: basic approaches", IEEE Robotics and Automation Magazine, 13(4):82-90, Dec. 2006.

[6] P. Corke, S. Hutchinson, "A new partitioned approach to imagebased visual servo control", IEEE Trans. on Robotics and Automation, 17(4):507-515, Aug. 2001.

[7] L. Deng, F. Janabi-Sharifi, W. Wilson, "Hybrid strategies for image constraints avoidance in visual servoing, IROSO2, Lausanne, pp 348353, Oct. 2002.

[8] B. Espiau, F. Chaumette, P. Rives, "A new approach to visual servoing in robotics", IEEE Trans. on Robotics and Automation, 8(3):313-326, June 1992.

[9] N. Gans, S. Hutchinson, P. Corke, "Performance tests for visual servo control systems, with application to partitioned approaches to visual servo control", Int. Journal of Robotics Research, 22:955-981, Oct. 2003.

[10] N. Gans, S. Hutchinson, "Stable visual servoing through hybrid switched-systems control, IEEE Trans. on Robotics, 23(3):530-540, June 2007.

[11] S. Hutchinson, G. Hager, P. Corke, "A tutorial on visual servo control", IEEE Trans. on Robotics and Automation, 12(5):651-670, Oct. 1996.

[12] F. Janabi-Sharifi, W. Wilson, "Automatic selection of image features for visual servoing", IEEE Trans. on Robotics and Automation 13(6):890-903, Dec. 1997.

[13] E. Malis, F. Chaumette, S. Boudet, "2 1/2 D visual servoing", IEEE Trans. on Robotics and Automation, 15(2):238-250, Apr. 1999.

[14] E. Malis, "Improving vision-based control using efficient second-order minimization techniques", ICRA'04, pp 1843-1848, New Orleans, Apr. 2004

[15] E. Marchand, F. Spindler, F. Chaumette, "ViSP for visual servoing: a generic software platform with a wide class of robot control skills", IEEE Robotics and Automation Magazine, vol. 12(4):40-52, Dec. 2005 ,

[16] P. Martinet, J. Gallice, D. Khadraoui, "Vision based control law using 3d visual features", Proc. WAC 96, pp. 497-502, Montpellier, May 1996.

[17] W. Wilson, C. Hulls, G. Bell, "Relative End-Effector Control Using Cartesian Position Based Visual Servoing", IEEE Trans. on Robotics and Automation, 12(5):684-696, Oct. 1996. 